This item was submitted to Loughborough's Research Repository by the author.

Items in Figshare are protected by copyright, with all rights reserved, unless otherwise indicated.

\title{
Experimental assessment of the effects of cross-traffic on Wi-Fi video
} streaming

PLEASE CITE THE PUBLISHED VERSION

http://dx.doi.org/10.1016/j.measurement.2011.06.012

PUBLISHER

(C) Elsevier

VERSION

AM (Accepted Manuscript)

LICENCE

CC BY-NC-ND 4.0

\section{REPOSITORY RECORD}

Angrisani, L., Konstantinos G. Kyriakopoulos, A. Napolitano, David J. Parish, M. Vadursi, and W.G. Whittow. 2019. "Experimental Assessment of the Effects of Cross-traffic on Wi-fi Video Streaming". figshare. https://hdl.handle.net/2134/9478. 
This item was submitted to Loughborough's Institutional Repository (https://dspace.lboro.ac.uk/) by the author and is made available under the following Creative Commons Licence conditions.

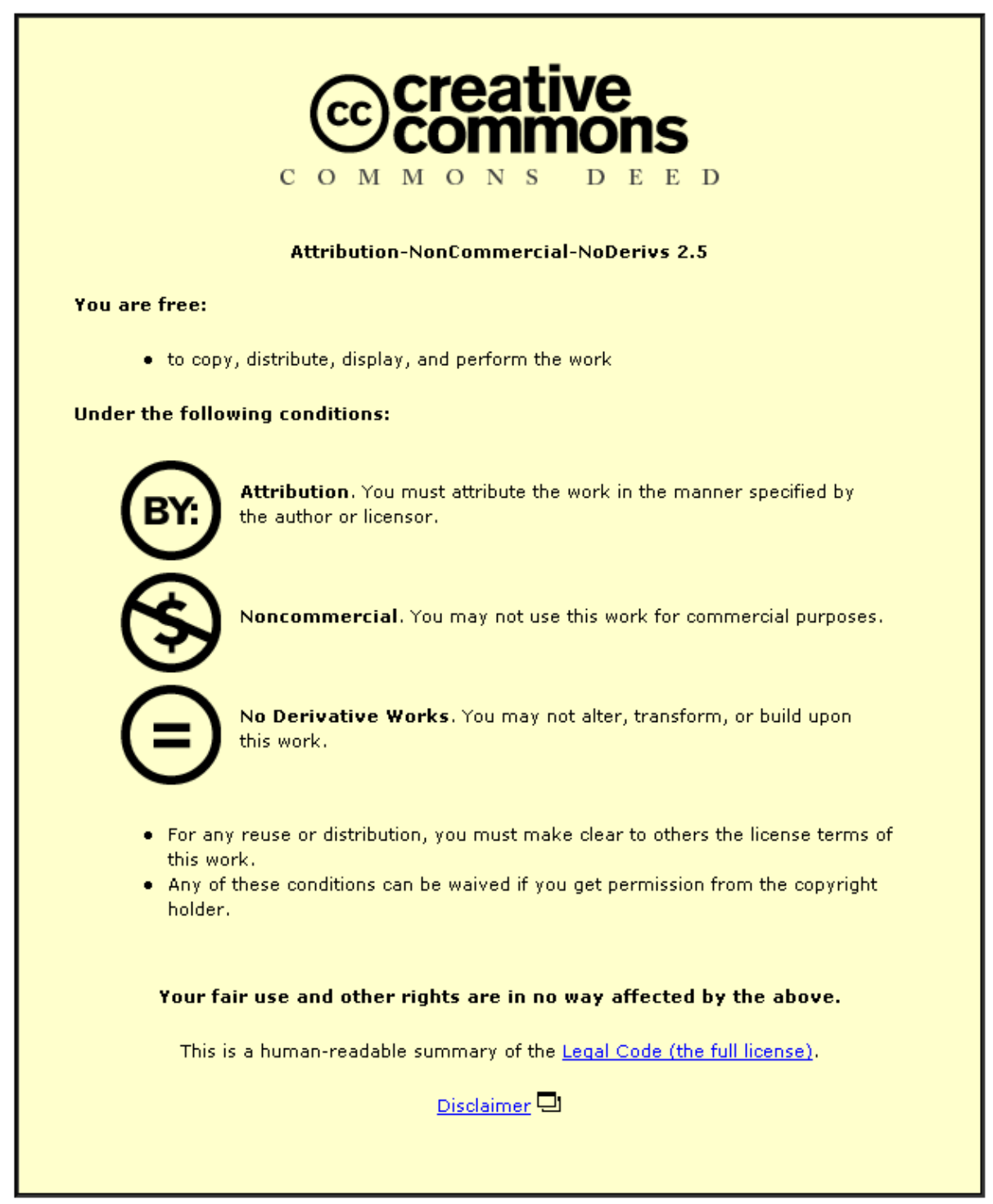

For the full text of this licence, please go to: http://creativecommons.org/licenses/by-nc-nd/2.5/ 


\title{
Experimental assessment of the effects of cross-traffic on Wi-Fi video streaming
}

\author{
Leopoldo Angrisani ${ }^{1, *}$, Konstantinos G. Kyriakopoulos ${ }^{2}$, \\ Aniello Napolitano ${ }^{3}$, David J. Parish ${ }^{2}$, \\ Michele Vadursi ${ }^{4}$, William G. Whittow ${ }^{2}$
}

${ }^{1}$ Dipartimento di Informatica e Sistemistica - Università degli Studi di Napoli Federico II Via Claudio, 21 - 80125 Napoli, Italy - Tel./Fax +390817683170

Email:angrisan@unina.it

${ }^{2}$ Department of Electronic and Electrical Engineering - Loughborough University Loughborough, LE11 3TU, U.K.

Email: \{elkk;d.j.parish;elwgw\}@lboro.ac.uk

${ }^{3}$ SESM s.c.a.r.l.

Via Circumvallazione Esterna, 116 - 80014 Giugliano in Campania (Napoli), Italy Email: anapolitano@sesm.it

${ }^{4}$ Dipartimento per le Tecnologie - Università degli Studi di Napoli "Parthenope" Centro Direzionale di Napoli, Isola C4 - 80143 Napoli, Italy

Email:michele.vadursi@uniparthenope.it

* corresponding author 


\section{EXPERIMENTAL ASSESSMENT OF THE EFFECTS OF CROSS-TRAFFIC ON WI-FI VIDEO STREAMING}

Abstract-Wi-Fi networks are the first and sometimes only choice for the video streaming in homes, airports, malls, public areas and museums. However, Wi-Fi networks are vulnerable to interference, noise and have bandwidth limitations. Due to the intrinsic vulnerability of the communication channel, and the large number of variables involved, simulation alone is not enough in the evaluation of the performance of wireless networks. Actually, there is a tendency to give experimental tests a central role in the assessment of Wi-Fi networks performance.

The paper presents an experimental analysis of the effects of cross traffic on the performance of video streaming over Wi-Fi, based on cross-layer measurements. Experiments are carried out in a semi-anechoic chamber, to prevent the results from being influenced by external factors. The experimental results permit to analyze the influence of cross traffic characteristics on cross layer measures and objective video quality metrics evaluated through a standardized approach.

Keywords: Wireless networks test and measurement, measurements for networking, Wi-Fi, video streaming, crosslayer measurements. 
Multimedia sharing, thanks to the progress of the video streaming technology underlying it, is rapidly spreading as an everyday practice. Nowadays, people want to access remotely stored videos from every part of their homes, on their own laptop or pocket PC. Access to multimedia contents via a PDA, a laptop or a pocket PC in airports, malls, public areas and museums is becoming more and more widespread, for entertainment purposes, public utility information, and ubiquitous commercial communication.

While being the first choice in home connectivity, thanks to their flexibility, low cost and quickness of installation, Wi-Fi networks, that are wireless local area networks (WLANs) based on IEEE 802.11 specifications, are the most practical technology solution for video streaming in public areas.

Despite such a huge development, the performance of Wi-Fi networks is sometimes hard to predict and to guarantee. This is mainly due to the poor stability and reliability of the radio link. In fact, while on wired channels signal integrity is assured by mechanical, electrical and protocol characteristics of the physical and data link layers [27], on wireless channels unpredictable and uncontrollable interference can severely affect data transmission, and ultimately degrade or even compromise the desired performance of the network [1]. The Wi-Fi standard exploits a scarce, shared, and noisy spectrum, i.e. the unlicensed 2.4 GHz Industrial Scientific Medical (ISM) band, on which other devices may operate simultaneously [2-6].

Performance evaluation based on simulations can be of help, but is not sufficient, due to the great number of variables involved. In such a direction, useful information can be achieved through ad-hoc laboratory and on-field measurements, exploiting proper test beds $[7,8]$. In the recent past, cross-layer measurements have come out to be a powerful option to assess and predict the performance of wireless and hybrid networks, as well as to troubleshoot them [9-16]. In the literature, a number of papers investigate on the feasibility of video streaming over Wi-Fi networks. In many cases, efficient solutions are proposed for improving the quality of video streaming. Nevertheless, only few of such contributions face the problem from an experimental point of view [15, 16]. Experimental performance assessment is, indeed, very important under critical conditions, e.g. when noise, in-channel interference and/or cross traffic are present. In such cases, in fact, the complexity of the protocol make it difficult to obtain reliable analytical and simulative results. 
Extensive standardisation related work has been undertaken on experimental measurement approaches for activities conducted at different layers of the network abstraction, however, these tend to be concentrated on a few layers only. For example, work has been undertaken to assess the quality of a video sequence at replay ( e.g. Mean Opinion Score [29]; VQM [26]), this is essentially an Application Layer issue. Separate standards relate to network performance measurement at the middle layers [30]; yet other independent approaches consider RF measurement at the Physical Layer [31]. However, to the best of the author's knowledge, little work has considered the simultaneous measurement of multiple performance metrics at different layers of the network. Hence this paper presents an approach to the measurement of the effects of interference at different layers on a Wireless network supporting video transfer for which no single coherent measurement standard applies.

Regarding Wi-Fi video streaming quality, a related work was presented in [21], where the video performance over WLAN is experimentally assessed with a cross-layer approach. The paper is interesting and investigates the impact of distance, possible obstacles and motion on the video quality, considering different metrics, including the peak signalto-noise ratio (PSNR), which is used as a quality indicator. It has to be noticed, however, that the choice of transmitting the cross traffic from the same source and to the same destination as with the video stream can raise some methodological concerns. In fact, more commonly bandwidth limitations are due to other hosts on the same wireless network rather than the same host, and the artificial cross traffic for the experiments should compete for the same wireless link, but should not at the same time represent an overhead for the host receiving the video, otherwise it could slow it down, and alter the results.

The authors have recently investigated the effects of Gaussian noise on the streaming time of the TCP (Transmission Control Protocol) connection [19]. However, UDP (User Datagram Protocol) is by far more adapt and widespread for wireless video streaming, due to its connectionless features. Thus, in this paper the attention is focused on the experimental analysis of Wi-Fi video streaming quality over UDP, with regard to both a normal UDP connection (hereinafter, referred to as normal mode) and a quality-of-service-oriented one ( $Q o S$ mode). In particular, the goal is to evaluate their performance in the presence of cross traffic. In fact, while the performance of Wi-Fi video streaming over UDP has already been studied with regard to noise and interference [17, 18], an experimental study based on cross-layer measurements, including VQM (Video Quality Metric) [26], in presence of cross traffic, is not reported in the literature. 
The paper is organized as follows: an overview of the IEEE 802.11x standards is given in Section II; the measurement test-bed, tools and procedure are respectively presented in Sections III; IV and V; experimental results are shown and discussed in Section VI; finally, conclusions are given in Section VII.

II.

\section{IEEE 802.11x STANDARDS}

The family of IEEE 802.11 standards concerns wireless connectivity for fixed, portable, and moving stations within a local area. It applies at the lowest two layers of the Open System Interconnection (OSI) protocol stack, namely the physical layer and data link layer.

The physical layer (PHY) essentially provides three functions. First, it interfaces the upper MAC sub-layer for transmission and reception of data. Second, it provides signal modulation through direct sequence spread spectrum (DSSS) techniques or orthogonal frequency division multiplexing (OFDM) schemes. Third, it sends a carrier sense indication back to the upper MAC sub-layer, to verify activity in the wireless channel. The data link layer includes the MAC sub-layer, which allows the reliable transmission of data from the upper layers over the PHY media. To this aim, it provides for a general controlled access to the shared wireless media, called carrier-sense multiple access with collision avoidance (CSMA/CA). It also protects the data being delivered through proper security policies. The IEEE 802.11 family currently includes multiple extensions to the original standard, which are based on the same basic protocol and are essentially different in terms of modulation techniques. The most popular extensions are those defined by the IEEE $802.11 \mathrm{a} / \mathrm{b} / \mathrm{g}$ amendments (also referred to as standards), on which most of today's manufactured devices are based. A further extension, namely IEEE 802.11e standard [20], has been emanated to support quality of service (QoS) in wireless environment. It defines specific MAC layer strategies to assure reliable performance on the wireless link for different traffic categories.

Nowadays, the IEEE $802.11 \mathrm{~g}$ standard is the most widely accepted worldwide. It involves the license-free $2.4 \mathrm{GHz}$ ISM band (2.4-2.4845 GHz), in the same way as the IEEE $802.11 \mathrm{~b}$ standard, and supports a maximum data rate of 54 Mbps, in the same way as the IEEE 802.11a standard. IEEE 802.11g standard devices are backwards compatible with IEEE $802.11 \mathrm{~b}$ ones. They use the OFDM modulation scheme for data rates of 6, 9, 12, 18, 24, 36, 48, and 54 Mbps, and revert to complementary code keying (CCK, as in the case of the IEEE $802.11 \mathrm{~b}$ standard) for 5.5 and $11 \mathrm{Mbps}$, and 
differential binary phase shift keying (DBPSK)/differential quadrature phase shift keying (DQPSK)+DSSS for 1 and 2 Mbps. Moreover, 14 different frequency channels are defined, each of which characterized by $22 \mathrm{MHz}$ bandwidth. In USA, channels 1 through 11 are allowed, in Europe channels 1 through 13 can be used, and in Japan only channel 14 is accessible. Due to the available bandwidth, the channels are partially overlapped, and the number of non-overlapping usable channels is only 3 in USA and Europe (e.g., channels 1, 6, and 11).

To assure QoS, the IEEE 802.11g standard refers to IEEE 802.11e one. With respect to the distributed coordination function (DCF), in fact, the IEEE 802.11e standard includes an additional hybrid coordination function (HCF), which both combines the capabilities of DCF and of the point coordination function (PCF) and adds some improvements. The HCF uses both a contention-based channel access method, called enhancement distributed channel access (EDCA) mechanism, for contention-based transfer, and a controlled channel access, referred to as controlled channel access (HCCA) mechanism, for contention-free transfer. In both mechanisms (EDCA and HCCA), the station is allowed to transmit only when it gains a transmission opportunity (TXOP), called EDCA TXOP or HCCA TXOP, respectively. In the EDCA mechanism, QoS is performed through the use of access categories (ACs), characterized by traffic categories (TC) and multiple independent backoff entities. In the IEEE 802.11e standard, a station is characterized by four ACs, having independent transmission queues. An AC is basically an enhanced variant of the DCF, which contends for a TXOP according to some suitable parameters [20].

In the HCCA mechanism, the access to the wireless medium is managed by using a hybrid coordinator (HC), whose access priority is higher than that of a station supporting QoS. The HC gains the control of the wireless medium waiting for a shorter time interval with respect to the stations using the EDCA procedures. In particular, when the wireless medium is assessed idle for at least one point inter-frame space (PIFS), it transmits the first frame with such a duration value as to cover the contention-free period. During this period, the $\mathrm{HC}$ assigns to the stations the needed TXOPs. At the end of either mechanism, an admission control phase is performed, as described in [20].

III. MEASUREMENT TEST-BED

Tests are conducted within a protected and controlled environment, i.e. a shielded semi-anechoic chamber compliant with electromagnetic compatibility requirements for radiated emission tests. Experiments aim to emulate the actual 
operating scenario of a WLAN compliant with IEEE 802.11g standard. A cross-layer approach is applied in order to assess the performance of WLAN in supporting video streaming applications. More specifically, different metrics at different protocol stack layers are measured: streaming time at application layer, lost packets at transport layer, and MAC retransmission at link layer. Additionally, video quality is measured through the software VQM, which implements a standardized method to objectively measure video quality [26].

A block diagram of the measurement test-bed is shown in Fig.1. It consists of the following components.

1) A WAP54G access point (AP) by Linksys, compliant with the IEEE 802.11g standard.

2) A notebook "Host1", with Intel Pentium Dual Core @ 1.73 GHz, 4 GB of RAM, which communicates with the AP at a 100 Mbps rate and through a $5 \mathrm{~m}$ length UTP category 3 cable, and acts as the source of the video stream.

3) Two notebooks, "Host2" and "Host3", which are equipped with Intel Core 2 Duo @ 2 GHz processor, 4 GB RAM and built-in Wi-Fi card (Airport) compliant with IEEE 802.11b/g standard, and act, respectively, as the receiver of the video stream and as the source of the cross traffic. They are both connected to the AP through an IEEE $802.11 \mathrm{~g}$ wireless connection, according to a DCF MAC layer access method along with CSMA/CA protocol. The parameters of the hosts are provided for the sake of completeness of the experimental setup description and for potential experiment repeatability purposes.

4) Two notebooks, "Host4" and "Host5", which are equipped with Intel Core 2 Duo @ 2 GHz processor and 4 GB RAM. Both notebooks have their built-in Wi-Fi card disabled, and are connected to an ASUS USB adapter Wi-Fi card, namely WL-167G, compliant with IEEE $802.11 \mathrm{~g}$ standard. They are thus used as monitoring devices for Host2 and Host3. 

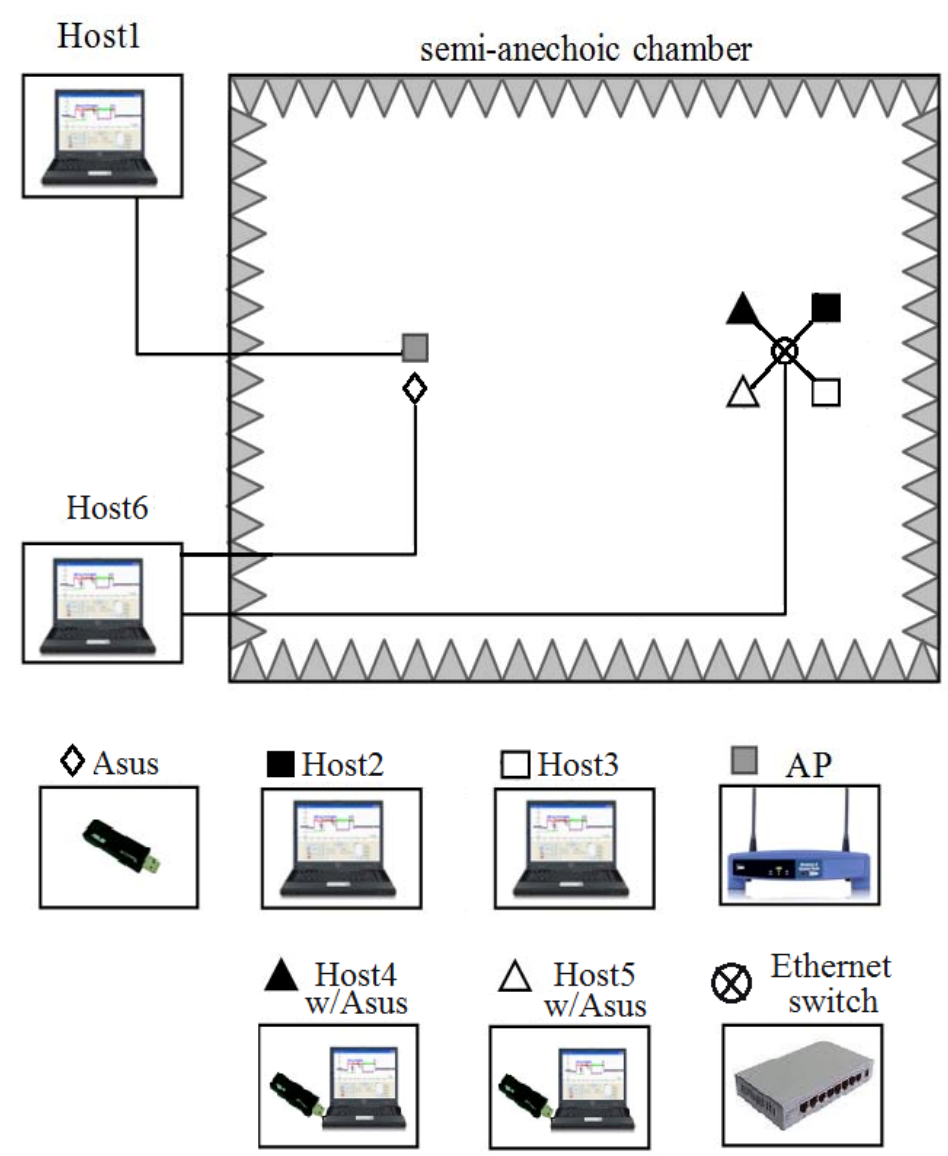

Fig. 1. Measurement testbed.

5) A further notebook, "Host6", which is connected to an Ethernet switch to which also Host2, Host3, Host4 and Host5 are connected. This makes it possible to control via remote desktop the four hosts in the chamber from Host6, which is outside.

6) Another ASUS WL-167G, which is placed near the AP connected to Host6 through a USB cable.

The same test-bed characterizes both the operating modes that have been considered for the experiments. DCF and HCF access methods are respectively exploited in the normal and $Q o S$ mode. Indeed, in the QoS mode, the AP is configured in such a way to recognize the video streaming packets as a traffic category, and applies the specified QoS, whereas, in the normal mode the best-effort service is invoked. 
The whole set of software tools used in the experiments are open-source, free available in the public domain. Specifically, VideoLAN [24] is a free cross-platform media player released under the GNU General Public License [28]. It can be used as a multicast and unicast streaming generator, supporting a large number of audio and video formats. It also allows the choice of the receiver buffer time length. In the experiments, VideoLAN is used by Host1 to generate the test videos, and by Host2 to decode the received data flow with a buffer time length of $300 \mathrm{~ms}$.

Wireshark is an open-source packet analyser tool for multilevel packet analysis [22]. It allows in-depth investigation about network problems and performance, and accurate testing of new protocols. It provides meaningful information about the incoming packets characteristics and contents. All information carried by packets is read, analysed and presented to the user by Wireshark. This include fields like timestamps, which indicate the time when a packet was recorded and flags in the packet indicating whether a packet has been retransmitted at the MAC layer or not. Packet losses are calculated by counting with Wireshark the number of packets sent by the sender and the number of packets received at the receiver. The number of lost packets can be calculated by subtracting the number of packets received from the number of packets sent. In the experiments, it is used both to assess the correct operation of the WLAN and measure the PLR.

D-ITG is a distributed Internet traffic generator [27], whose architecture allows the generation of traffic and the regulation of key parameters such as packet inter-departure time and length. It also allows measuring several QoS parameters at both sender and receiver side, and obtaining a complete report of measured parameters over the whole measurement time. D-ITG is, in particular, used to generate WLAN traffic in the second scenario.

VQM is a Video Quality Metric algorithm, based on the models referred to by ITU Recommendation BT.1683 [26]. It provides video quality estimates rather close to those achievable from subjective analysis. It requires two input video streams: the original one, taken as reference, and the effectively displayed one, possibly corrupted, to be analyzed. As final result, VQM provides an overall quality score, mapped on a scale from 0 up to 1 , where 0 means that no impairment is perceivable and 1 that a maximum level of impairment is visible. 
Experiments are conducted emulating a real-world unidirectional video streaming, compliant with H.264, and characterized by different video data rate. The features of the video used in the tests are chosen according to a typical configuration exploited for sharing and spreading video contents over the Internet. In particular, the video is characterized by, (i) Constrained Baseline profile (CBP), (ii) level two, (iii) CIF (Common Intermediate Format, $352 \times$ 288) picture format, (iv) three different mean data rate respectively of nearly $300 \mathrm{kbit} / \mathrm{s}$ (referred to as "low" profile), $600 \mathrm{kbit} / \mathrm{s}$ (referred as "medium" profile) and $800 \mathrm{kbit} / \mathrm{s}$ (referred as "high" profile), and (v) RTP/UDP as communication protocol.

Concurrently to the video streaming, a suitable cross traffic is generated on the same wireless link, in both modes, by synthetic traffic generator D-ITG. The cross traffic profile is CBR (constant bit rate), with bit rates equal either to $20 \mathrm{Mbps}$ or $40 \mathrm{Mbps}$ in order to encompass different conditions of network load. Furthermore, different IP cross traffic packet size is chosen, respectively equal to 512 bytes and 1400 bytes. Experiments with no cross traffic are also carried out.

Generally, Host2 generates a request to Host1, through the AP by wireless connection, for streaming an H.264 encoded video located on Host 1 . Once the connection is accepted, Host1 sends the video to Host2 via the AP, exploiting a UDP connection. Host2 down-streams the received H.264 video using the VLC media player.

The sniffer software Wireshark is installed on Host4, Host5 and Host6 in order to process the data captured by WL167G cards. The choice of having monitoring hosts which are different from those involved with the WLAN permits to gather packets retransmissions at MAC layer. Otherwise, they could not be captured from the machine that is generating the retransmissions neither from the machine that the retransmissions are destined to.

A cross-layer approach is applied in order to assess the performance of WLAN in supporting video streaming applications in presence/absence of QoS mechanisms. More specifically, different metrics at different protocol stack layers are measured, such as streaming time at application layer, lost packets at transport layer, and MAC retransmission at link layer. The streaming time is measured from the log file of the client computer as the difference of the timestamp of the last UDP video packet from the timestamp of the first UDP video packet. The number of 
MAC retransmissions at link layer and the packet loss ratio at transport layer are jointly measured, because they give different information: the former gives an indication of the relative data link layer conditions and can be significantly different from zero even when no packet is lost at transport layer. Additionally, video quality is measured through the software VQM, which implements a standardized method to objectively measure video quality in off-line mode. In detail, the streamed video is stored by VLC on the client after each experiment, and then compared by VQM to the original video file, which acts as reference.

VI.

\section{EXPERIMENTAL RESULTS}

\section{A. Normal mode scenario}

Table I synthesizes the results of 15 experiments related to a normal mode UDP connection. Experiments are first ordered by cross traffic bit rate, then by cross traffic packet size and, finally, by video bit rate. Fig. 2 to 4 respectively depict packet loss ratio (PLR), number of MAC-layer retransmissions (Retx) and time to stream results.

The first three experiments show that when the video streaming quality (VQM) is almost perfect no cross traffic is present, as none of the packets is lost. Although these tests could seem banal, it has been done to verify that the connection is working properly, as so is the application.

Table I. Measurement results in the normal mode scenario

\begin{tabular}{|c|c|c|c|c|c|c|c|c|}
\hline \multirow{2}{*}{ \# } & \multicolumn{2}{|c|}{ Cross traffic } & \multirow{2}{*}{$\begin{array}{l}\text { Video } \\
\text { profile }\end{array}$} & \multicolumn{5}{|c|}{ Measurement results } \\
\hline & $\begin{array}{c}\text { Packet size } \\
\text { [byte] }\end{array}$ & $\begin{array}{l}\text { Bit rate } \\
\text { [Mbit/s] }\end{array}$ & & Packets sent & Retransmissions & $P L R[\%]$ & $\begin{array}{l}\text { Time to } \\
\text { stream [s] }\end{array}$ & $\begin{array}{l}\text { VQM } \\
\text { score }\end{array}$ \\
\hline 1 & 0 & 0 & Low & 5276 & 1 & 0 & 116.7 & 0 \\
\hline 2 & 0 & 0 & Medium & 6192 & 5 & 0 & 112.9 & 0 \\
\hline 3 & 0 & 0 & High & 6617 & 5 & 0 & 135.1 & 0 \\
\hline 4 & 1400 & 20 & Low & 5237 & 3666 & 0 & 157.1 & 0.1 \\
\hline 5 & 1400 & 20 & Med & 6047 & 4033 & 0 & 114.2 & 0.1 \\
\hline 6 & 1400 & 20 & High & 6519 & 3817 & 0 & 155.5 & 0.1 \\
\hline 7 & 512 & 20 & Low & 4152 & 2354 & 21.8 & 112.8 & 0.4 \\
\hline 8 & 512 & 20 & Med & 6034 & 3503 & 20.4 & 128.2 & 0.4 \\
\hline 9 & 512 & 20 & High & 6468 & 3825 & 20.3 & 141.3 & 0.4 \\
\hline
\end{tabular}




\begin{tabular}{|l|l|l|l|l|l|l|l|l|}
\hline 10 & 1400 & 40 & Low & 5105 & 2111 & 28.5 & 152.4 & 0.6 \\
\hline 11 & 1400 & 40 & Med & 6027 & 2131 & 27.6 & 140.5 & 0.6 \\
\hline 12 & 1400 & 40 & High & 6459 & 2309 & 26.9 & 164.5 & 0.6 \\
\hline 13 & 512 & 40 & Low & 5347 & 1367 & 55.2 & 137.2 & 0.8 \\
\hline 14 & 512 & 40 & Med & 6144 & 1272 & 50.3 & 119.2 & 0.8 \\
\hline 15 & 512 & 40 & High & 6412 & 1306 & 50.9 & 143.8 & 0.8 \\
\hline
\end{tabular}

There is a clear influence of cross traffic rate on the performance of the video streaming and a direct relation between PLR and cross traffic bit rate. Fig. 2 clearly shows this trend: the higher the cross traffic rate, the higher (on average) the number of packets that do not reach the receiver due to congestion. Given that the PLR is the ratio of packets received over packets transmitted, the PLR increases with increasing cross traffic rate. However, contrary to what one would reasonably expect, another metric that is the number of retransmissions (see Fig. 3), seem to prove such consideration wrong. The experimental outcomes show that number of retransmission is almost halved when the bit rate doubles from $20 \mathrm{Mbits} / \mathrm{s}$ to $40 \mathrm{Mbit} / \mathrm{s}$. In fact, looking at the number of retransmissions while forgetting about the PLR is misleading, but thanks to the cross-layer approach such a paradox can be explained as follows. When the wireless link becomes more congested, more video packets are lost because they are dropped by the AP. As retransmissions are counted at MAC layer, their number decreases because less video packets are transmitted on the wireless link.The dependence of PLR on cross traffic packet size can also be simply explained. Given the cross traffic bit rate, smaller packets mean a higher number of total packets and therefore, according to the MAC layer protocol, a higher overhead which causes the channel to be occupied for a larger proportion of time. Thus, more video packets are dropped in the queue of the AP and, consequently, lost. Therefore, with smaller cross traffic packet size, the PLR increases.

Finally, it is interesting to observe that while the PLR is extremely sensitive to cross traffic, the streaming time seems to vary randomly but without significant correlation to it. Such a behavior is peculiar to UDP: the video source host emits packets at the given rate, then these packets have to share the channel with cross traffic and a number of them is possibly dropped by the AP. However, being UDP a connectionless protocol, none of these packets is retransmitted at transport layer and, so, the streaming time does not vary significantly. On the contrary, it is the quality 
of the video that is affected and reflects the packet loss. Had TCP been used, a quick degradation of the streaming time would have been observed as the cross traffic increased, as already noticed with noise [19].

It is interesting to observe that, in line of principle, a relation between the cross traffic and video quality could be exploited by the network for extracting information about video quality, on the basis of the actual amount of data traffic present in the network. A proper control strategy of data traffic flow could thus be implemented to assure a defined quality to video steaming applications. Similarly, the relation between VQM and PLR can allow the video receiver to implement a suitable feedback procedure towards the AP for reducing the data traffic into the network as long as the desired video quality is achieved.

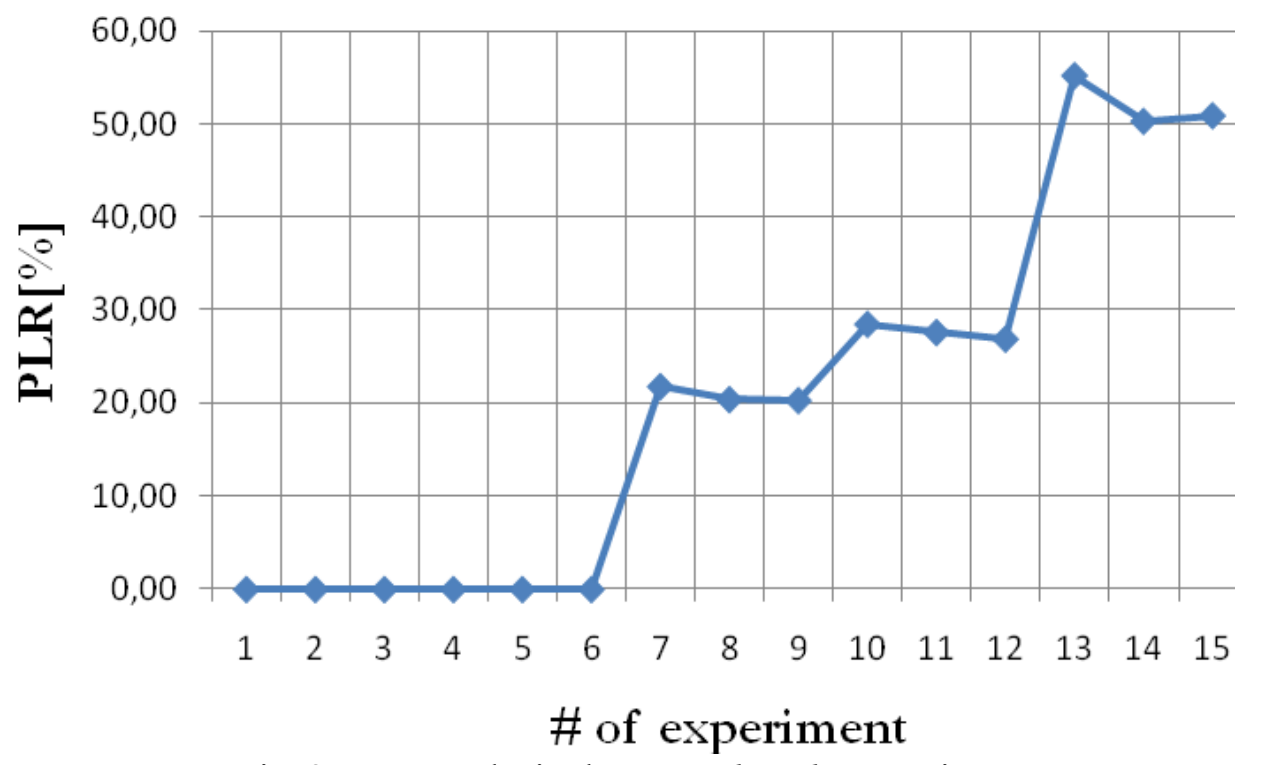

Fig. 2. PLR results in the normal mode scenario. 


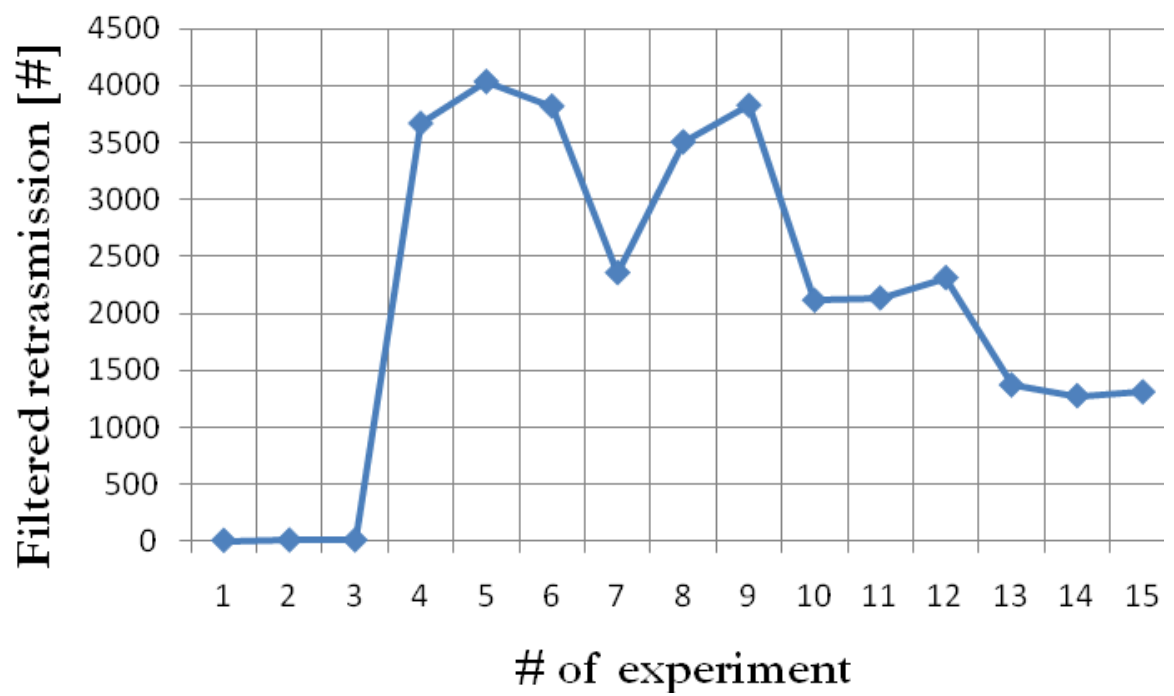

Fig. 3. Retransmissions results in the normal mode scenario.

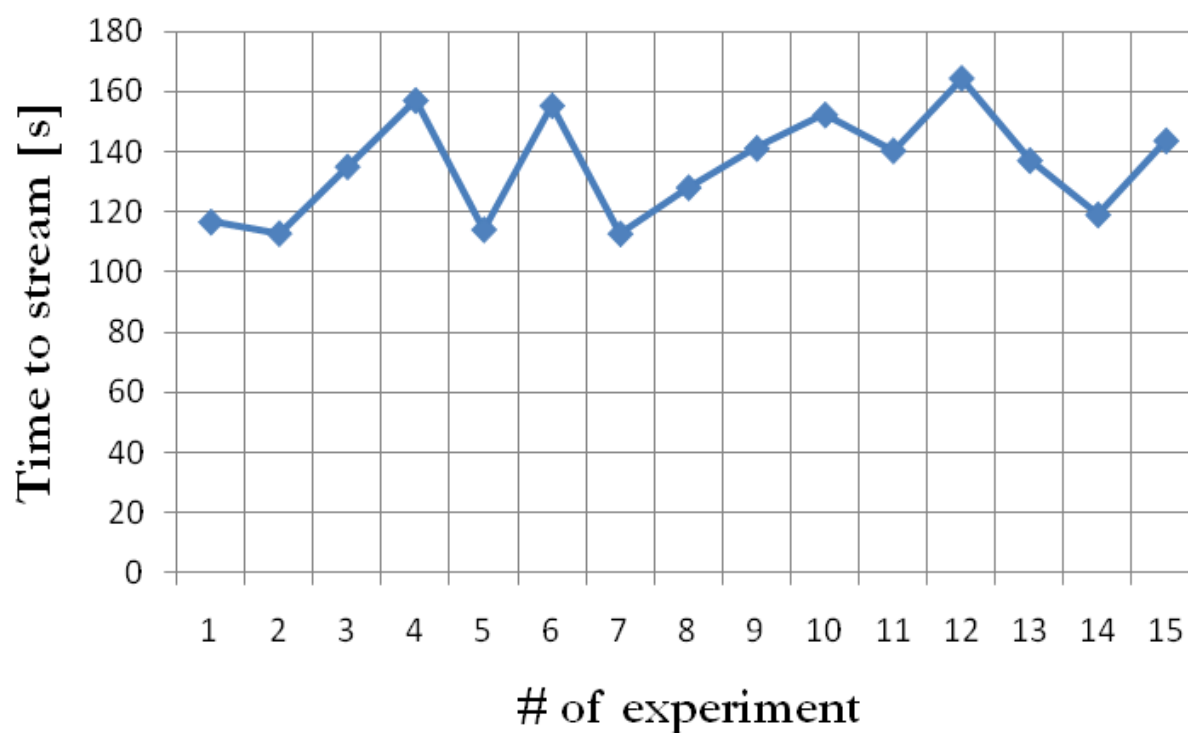

Fig. 4. Streaming time results in the normal mode scenario. 


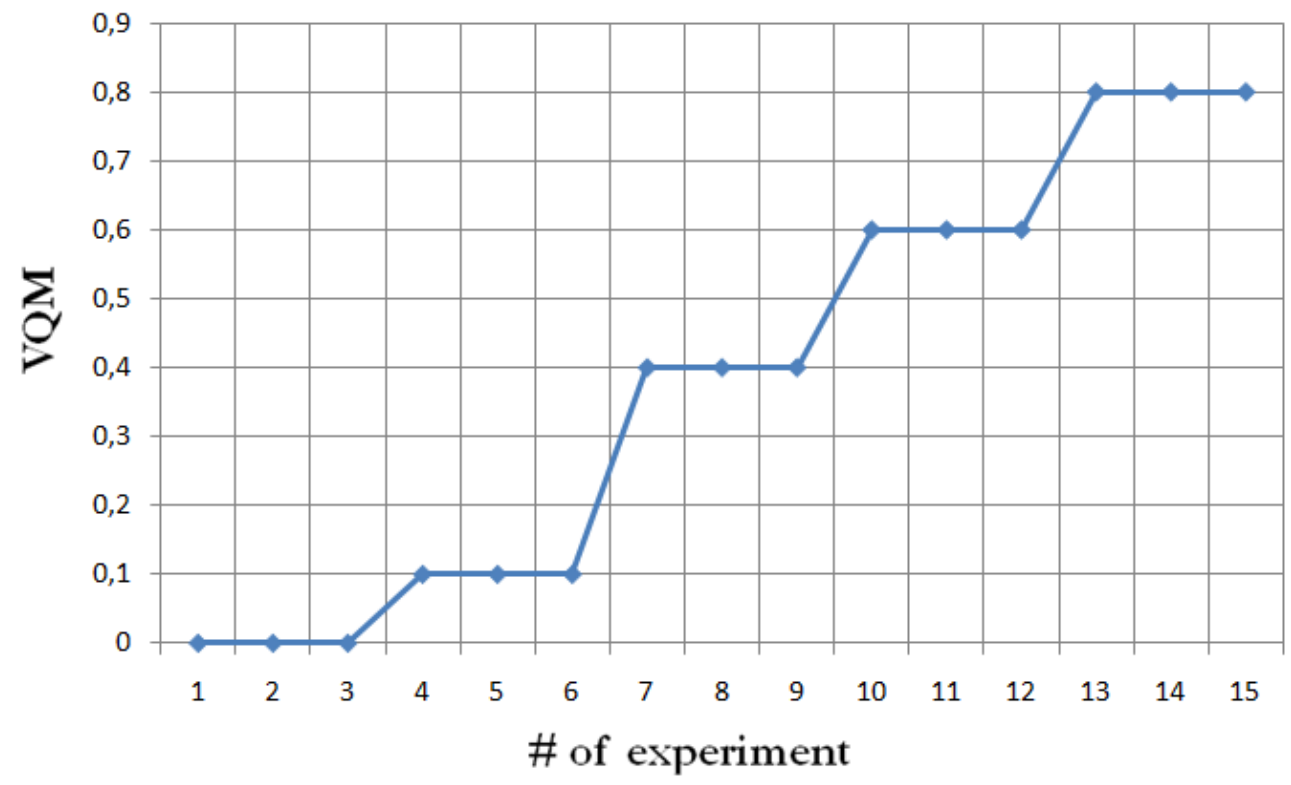

Fig. 5. VQM results in the normal mode scenario.

\section{B. QoS mode scenario}

As already discussed, in the QoS mode scenario, the WLAN has suitable been configured for providing QoS by giving precedence to video streaming packets with respect to cross traffic packets. More specifically, the AP has been configured in such a way as to classify the video streaming packets as a traffic category (i.e. AC_VI), through proper rules, and supply the QoS according with the following parameters: i) TXOP equal to $3008 \mathrm{~ms}$; $\mathrm{CW}_{\min }=15$; and $\mathrm{CW}_{\max }$ equal to 31 . Being these values suggested by IEEE 802.11e standard for AC_VI category, they are also given as default ones into any AP setup configuration.

The obtained results, in terms of PLR, VQM score, time to stream and retransmissions, are summarized in Table II for all the experiments. Fig. 6 to 9 separately show the metrics taken into consideration.

From the measurement results, the following considerations can be made.

- VQM score is strictly related to PLR. Indeed, only when a heavy PLR is experienced the video quality worsens. This is the case of experiment number 15. On the contrary, if low PLR values are measured, the final quality falls into an acceptable value range. 
- There is a non linear relationship between cross traffic (both rate and packet size) and PLR as seen in Fig. 7. Initially, PLR remains very small (PLR $\leq 1.3 \%$ ) as the cross traffic is not high enough to create congestion in the link and, therefore, there are no lost packets. However, if a threshold is exceeded, as happens in point 15 of Fig. 7 with PLR $=4.0 \%$, then the effects of cross traffic on PLR increase exponentially.

- The HCF mechanism improves the channel access function reducing the nominal values of all metrics except the time to stream. This means the QoS mechanism assures high priority level to video streaming also in presence of cross traffic characterized by a high bit rate.

- The time to stream does not benefit from the QoS mechanism. In fact, it is still distributed in a random way, in agreement with what observed in the normal mode scenario. This is reasonable because the packet loss is due only to buffer overflow, as no air collisions can be experienced in our test-bed.

- It is interesting to observe that high time to stream values (as measured for experiment number 15) do not influence the final video quality score. That is, the buffer length of VLC is capable of managing these deviations and providing a good video quality to the final users.

Table II. Measurement results in the QoS mode scenario.

\begin{tabular}{|c|c|c|c|c|c|c|c|c|}
\hline \multirow{2}{*}{$\#$} & \multicolumn{2}{|c|}{ Cross traffic } & \multirow{2}{*}{$\begin{array}{c}\text { Video } \\
\text { profile }\end{array}$} & \multicolumn{5}{|c|}{ Measurement results } \\
\cline { 2 - 5 } & $\begin{array}{c}\text { Packet size } \\
{[\text { byte] }}\end{array}$ & $\begin{array}{c}\text { Bit rate } \\
{[\text { Mbit/s] }}\end{array}$ & & Packets sent & Retransmissions & PLR[\%] & $\begin{array}{c}\text { Time to } \\
\text { stream [s] }\end{array}$ & $\begin{array}{c}\text { VQM } \\
\text { score }\end{array}$ \\
\hline 1 & 0 & 0 & Low & 5276 & 1 & 0 & 116.7 & 0 \\
\hline 2 & 0 & 0 & Med & 6192 & 2 & 0 & 112.9 & 0 \\
\hline 3 & 0 & 0 & High & 6617 & 1 & 0 & 114.1 & 0 \\
\hline 4 & 1400 & 20 & Low & 5237 & 100 & 0 & 120.1 & 0.1 \\
\hline 5 & 1400 & 20 & Med & 6047 & 107 & 0 & 144.2 & 0.1 \\
\hline 6 & 1400 & 20 & High & 6519 & 108 & 0 & 143.5 & 0.1 \\
\hline 7 & 512 & 20 & Low & 4152 & 89 & 0 & 138.6 & 0 \\
\hline 8 & 512 & 20 & Med & 6034 & 78 & 1.0 & 146.2 & 0.2 \\
\hline 9 & 512 & 20 & High & 6468 & 120 & 1.2 & 141.3 & 0.1 \\
\hline 10 & 1400 & 40 & Low & 5105 & 75 & 0 & 142.0 & 0.1 \\
\hline 11 & 1400 & 40 & Med & 6027 & 111 & 0.6 & 138.9 & 0.1 \\
\hline 12 & 1400 & 40 & High & 6459 & 160 & 1.0 & 141.6 & 0.2 \\
\hline 13 & 512 & 40 & Low & 5347 & 101 & 0 & 129.6 & 0 \\
\hline 14 & 512 & 40 & Med & 6144 & 131 & 1.3 & 135.2 & 0.1 \\
\hline 15 & 512 & 40 & High & 6412 & 260 & 4.0 & 147.2 & 0.4 \\
\hline
\end{tabular}




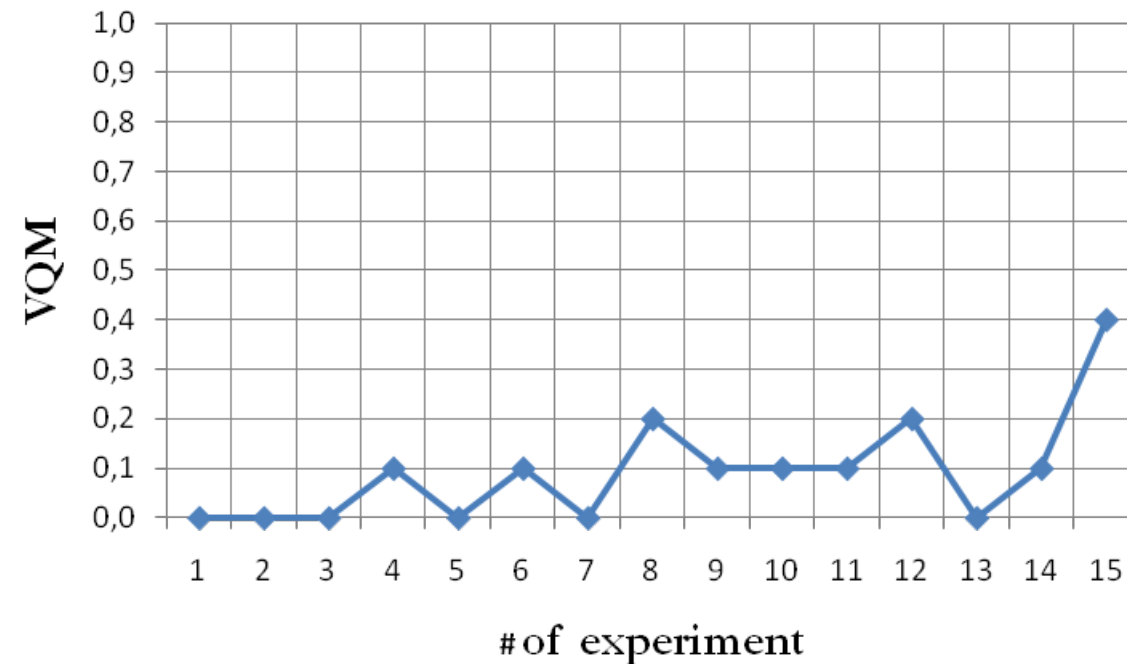

Fig. 6. VQM results in the $Q o S$ mode scenario.

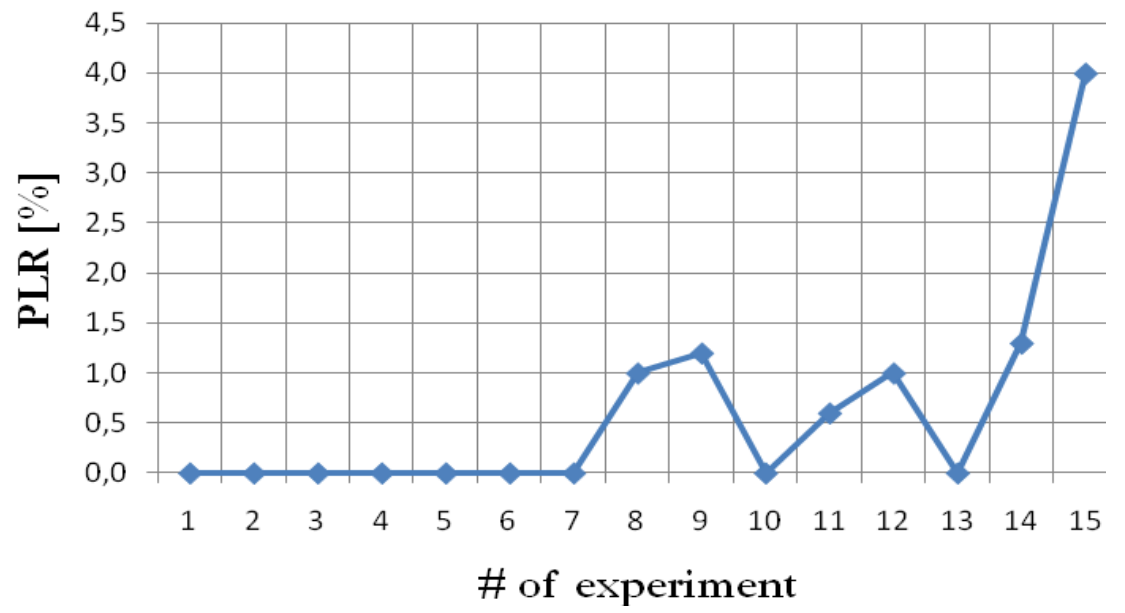

Fig. 7. PLR results in the $Q o S$ mode scenario. 


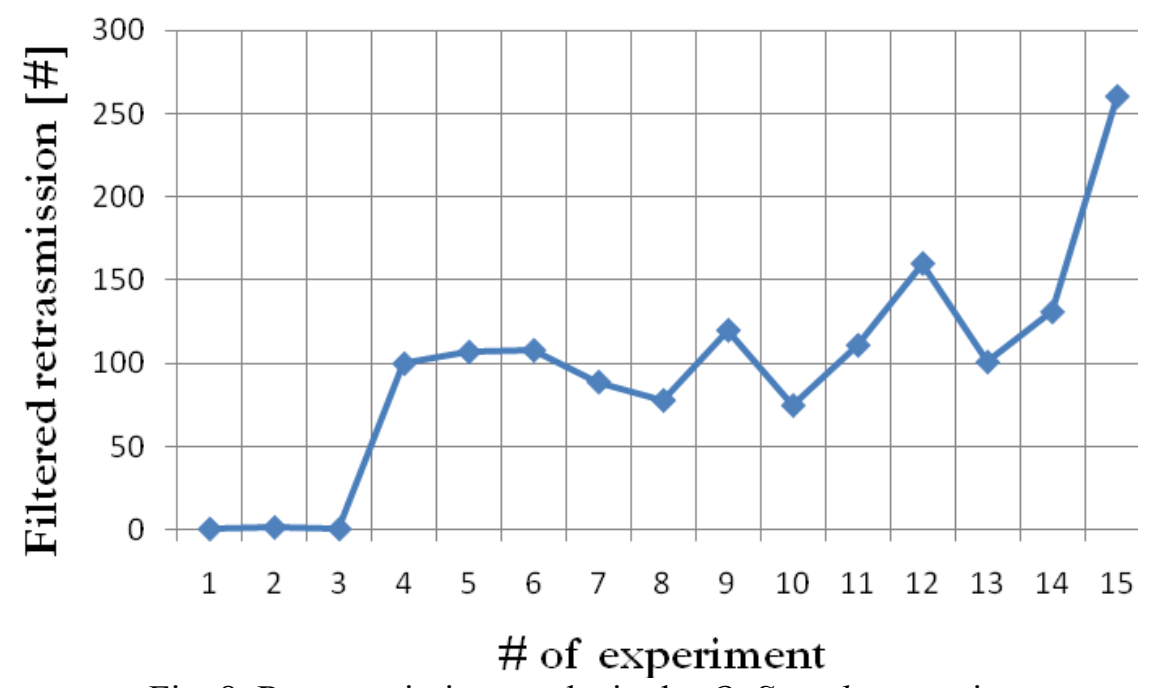

Fig. 8. Retransmission results in the $Q o S$ mode scenario.

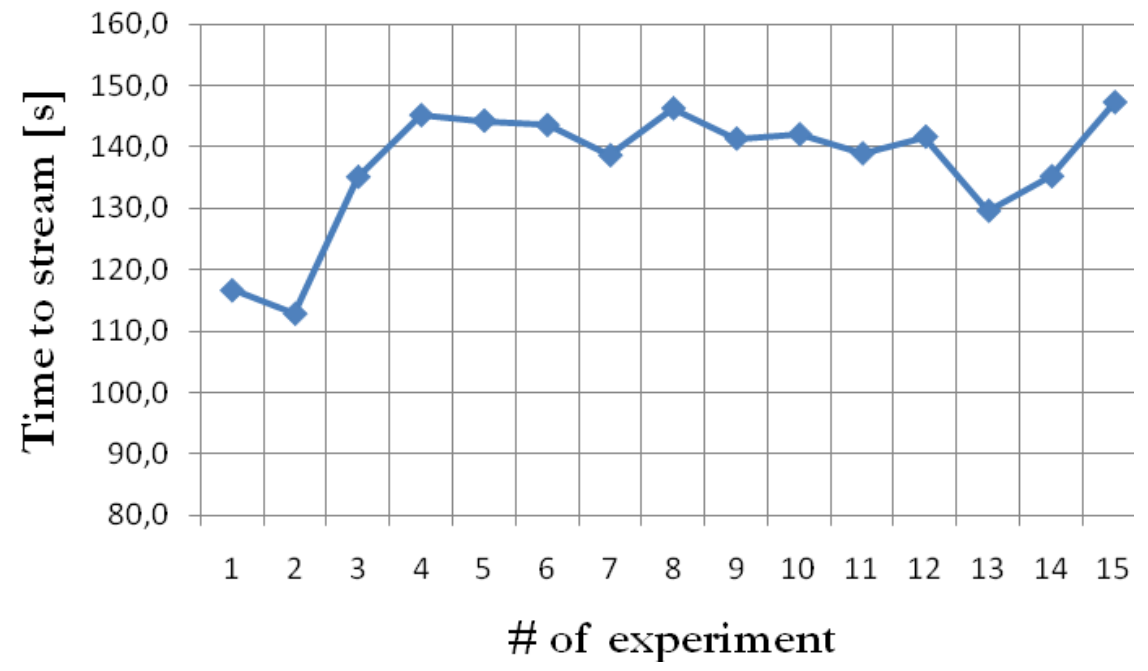

Fig. 9. Time to stream results in the QoS mode scenario.

VII.

CONCLUSION

The paper has presented an experimental study of the effects of bandwidth limitations on video streaming over WiFi. A proper measurement test-bed has been designed for the scope in a controlled environment, i.e. a semianechoic chamber. The transport layer protocol considered is UDP, which is by far the more used for video streaming over WiFi. Tests have been conducted in two operating modes: a normal mode and a QoS mode. 
Experimental results have shown a dependence of PLR on the cross traffic rate and packet size, but there is no evidence of a dependence of the total streaming time on the cross traffic. This is reasonable considering that UDP is a connectionless protocol. Regarding VQM, it has been observed that high time to stream values does not influence the final video quality score. That is, the buffer length of VLC is capable of managing these deviations and providing a good video quality to the final users. Moreover, it has come out that only when a heavy PLR is experienced the video quality worsens significantly. On the contrary, if low PLR values are measured, the final quality falls into an acceptable value range.

Ongoing research activity is focused on measuring wireless video streaming performance under different QoS assurance strategies, as well as considering more complex scenarios where cross traffic and in-channel interference are jointly affecting the communication.

\section{REFERENCES}

[1] A. P. Jardosh, K. N. Ramachandran, K. C. Almeroth, E. M. Belding-Royer, ”Understanding Congestion in IEEE 802.11b Wireless Networks”, Proc. of Internet Measurement Conference IMC 2005, pp. 279-292.

[2] M. van der Schaar, Y. Andreopoulos, Z. Hu, "Optimized scalable video streaming over IEEE 802.11 a/e HCCA wireless networks under delay constraints," IEEE Trans. Mobile Comput., vol. 5, no. 6, pp. 755-768, June 2006.

[3] D. Majumdar, G. Sachs, I.V. Kozintsev, K. Ramchandran, "Multicast and Unicast Real-Time Video Streaming over Wireless LANs," IEEE Trans. Circuits and Systems for Video Technology, vol. 12, no. 6, pp. 524-534, June 2002.

[4] J. Kuri and S.K. Kasera, "Reliable multicast in multi-access wireless LANs", Wireless Networks, Vol. 7, No. 4, pp. 359-369, July 2001.

[5] I. Haratcherev, J. T. K. Langendoen, R. Lagendijk, and H. Sips, "Optimized Video Streaming over 802.11 by Cross-Layer Signaling", IEEE Communications Magazine, January 2006.

[6] K. Balachandran, S. R. Kadaba, and S. Nanda, "Channel Quality Estimation and Rate Adaptation for Cellular Mobile Radio," IEEE JSAC, vol. 17, no. 7, July 1999, pp. 1244-56. 
[7] L. Angrisani, S. D'Antonio, M. Esposito, M. Vadursi, “Techniques for available bandwidth measurement in IP networks: a performance comparison, Computer Networks, vol. 50, No. 3, pp. 332-349, 22 Feb 2006.

[8] G. Aceto, A. Botta, A. Pescape, M. D'Arienzo, "UANM: a platform for experimenting with available bandwidth estimation tools," Proc. of IEEE Symp. on Computers and Communications (ISCC), pp. 174-179, 2010.

[9] T. K. Chiew, P. Ferre, D. Agrafiotis, A. Molina, A.R. Nix, D.R. Bull, "Cross-layer WLAN measurement and link analysis for low latency error resilient wireless video transmission," Proc. of Intern. Conf. on Consumer Electronics, ICCE 2005, pp.177-178 8-12 Jan. 2005.

[10] A.Valera, P.W.Q. Lee, H.-P. Tan, W.K.G. Seah, Zhi Ang Eu, “An in-situ measurement approach for IEEE 802.11 wireless multihop networks, Proc. of Instrum. and Meas. Tech. Conf., 2009_ I2MTC 2009, Singapore, 5-7 May 2009.

[11] L. Angrisani, A. Pescapè, G. Ventre, M. Vadursi, "Performance measurement of IEEE 802.11b-based networks affected by narrowband interference through cross-layer measurements," IET Communications, vol. 2, No.1, Jan. 2008, pp. 82-91.

[12] L. Angrisani, M. Vadursi, "Cross-Layer Measurements for a Comprehensive Characterization of Wireless Networks in the Presence of Interference," IEEE Trans. on Instrumentation and Measurement, vol.56, No.4, Aug. 2007, pp.1148-1156.

[13] K. G. Kyriakopoulos, W. Whittow, D. J. Parish, "A Framework for Cross-Layer Measurements in Wireless Networks", Proc. of the Fifth IEEE Advanced Inter-national Conference on Telecommunications (AICT 2009) part of WebTel 2009, Venice, Italy, 24-28 May 2009.

[14] R. Li, D. J. Parish, K. G. Kyriakopoulos, "A Framework for Cross-layer Measurement of 3G and Wi-Fi Combined Networks", Proc. of PGNet 2008: The $9^{\text {th }}$ Annual Post Graduate Symposium on The Convergence of Telecommunications, Networking \& Broadcasting, Liverpool, U.K., 23-24 June 2008.

[15] A. Sheth, C. Doerr, D. Grunwald, R. Han, D. Sicker, "MOJO: A Distributed Physical Layer Anomaly Detection System for 802.11 WLANs,” Proc. of MobiSys'06, June 19-22, 2006, pp. 191-204. 
[16] J. Yeo, M. Youssef, T. Henderson, A. Agrawala, "An Accurate Technique for Measuring the Wireless Side of Wireless Networks," Proc of the Intern. Workshop on Wireless Traffic Measurements and Modelling WiTMeMo 2005, pp. 13-18.

[17] L. Angrisani, A. Napolitano, A. Sona, “Are IEEE 802.11 Wireless Networks Appropriate for Video Streaming in the Presence of Interference? An Experimental Answer," Proc. of I²MTC 2008, IEEE Int. Instr. and Meas. Tech. Conf., Victoria, Canada, May 12-15, 2008 pp.1722 - 1727.

[18] L. Angrisani, A. Napolitano, A. Sona, "Cross-Layer Measurement on an IEEE 802.11g Wireless Network Supporting MPEG-2 Video Streaming Applications in the Presence of Interference," EURASIP Journal on Wireless Communications and Networking, vol. 2010, 2010.

[19] L. Angrisani, K. Kyriakopoulos, A. Napolitano, D. J. Parish, M. Vadursi, W. Whittow, “An experimental analysis of the effects of noise on Wi-Fi video streaming," Proc. of the IEEE Intern. Instr. and Meas. Tech. Conf. 2010, I²MTC 2010, Austin (USA), 3-6- May 2010.

[20] IEEE Standard for Information technology--Telecommunications and information exchange between systemsLocal and metropolitan area networks-Specific requirements Part 11: Wireless LAN Medium Access Control (MAC) and Physical Layer (PHY) specifications: Amendment 8: Medium Access Control (MAC) Quality.

[21] Y. Ennaji, M. Boulfmalf, C. Alaoui, “Experimental Analysis of Video Performance over Wireless Local Area Networks,” Proc. of Intern. Conf. on Multimedia Computing and Systems ICMCS ’09, Morocco, 2-4 April 2009. [22] http://www.wireshark.org [online].

[23] J. F. Kurose, K. W. Ross, "Computer Networking: A Top-Down Approach Featuring the Internet", Addison Wesley, 2003.

[24] http://www.videolan.org/vlc/ [online].

[25] ITU-T, Recommendation H.264 - Advanced video coding for generic audiovisual services http://www.itu.int/rec/ dologin pub.asp?lang=e\&id=T-REC-H.264-201003-I!!PDF-E\&type=items [online]. 
[26] ITU-R BT.1683, “Objective perceptual video quality measurement techniques for standard definition digital broadcast television in the presence of a full reference", Jan. 2004.

[27]http://www.grid.unina.it/software/ITG/ [online]

[28]http://www.gnu.org/licenses/gpl.html [online]

[29] ITU-R BT.500-11. 2002. Methodology for the subjective assessment of the quality of television pictures.

[30] http://www.rfc-editor.org/in-notes/rfc2544.txt (accessed 23/03/11).

[31]T. Alexander, "Optimizing and Testing WLANs: Proven Techniques for Maximum Performance" Chapter 4, pp 75-105, Newnes, 2007 